
\title{
25 Research Soure \\ Comparison of Sagittal Spinal Alignment on Standing Plain X-Rays and Supine MRI in Degenerative Lumbar Disease
}

\section{Yulu Wang}

Zhejiang University School of Medicine Second Affiliated Hospital https://orcid.org/0000-0003-17230227

\section{Jinwu Bai}

Zhejiang University School of Medicine Second Affiliated Hospital

\section{Zhijun Pan}

Zhejiang University School of Medicine Second Affiliate Hospital

Deting Xue ( $\nabla$ blueskine@zju.edu.cn )

\section{Research article}

Keywords: sagittal spinal alignment, standing plain X-rays, supine MRI, degenerative lumbar disease

Posted Date: May 12th, 2020

DOI: https://doi.org/10.21203/rs.3.rs-26627/v1

License: (1) This work is licensed under a Creative Commons Attribution 4.0 International License. Read Full License 


\section{Abstract}

Background:The purpose of present study is to examine the possible correlation between standing plain X-rays and supine MRI for evaluating spinal sagittal alignment in degenerative lumbar disease.

Methods: The characteristics and images of 64 patients with degenerative lumbar diseases were reviewed retrospectively. The thoracolumbar junction kyphosis (TJK), lumbar lordosis (LL), and sacral slope (SS) were measured on lateral plain X-rays and by magnetic resonance imaging (MRI). Inter- and intra-observer reliability were tested using intra-class correlation coefficients.

Results: The results suggested that TJK measurements obtained from MRI tended to underestimate the radiographic measures by $2^{\circ}$, whereas SS measurements obtained from MRI tended to overestimate the radiographic measures by $2^{\circ}$. The LL measurements obtained from MRI were approximately equal to the radiographic measures. The X-ray and MRI measurements were linearly related.

Conclusions:In conclusion, supine MRI can be directly translated into sagittal alignment angle measurements obtained from standing X-rays with an acceptable degree of accuracy. This can avoid the impaired view caused by the overlapping ilium, while reducing the patient's exposure to radiation.

\section{Introduction}

Spinal alignment, which includes coronal and sagittal alignment, is an important part of preoperative planning and surgical reconstruction in spinal surgery [1]. In degenerative lumbar diseases, coronal alignment reconstruction is an important issue for degenerative lumbar scoliosis, whereas sagittal alignment is usually considered in spondylolisthesis and loss of lumbar lordosis [2]. The standing X-ray is considered the gold standard to measure spinal alignment [3]; however, repeated exposure of patients to high radiation doses increases the risk of developing health problems $[4,5]$. In addition, the lateral radiological assessment of the lumbosacral region is usually obscured by the overlapping ilium.

Magnetic resonance imaging (MRI) does not involve radiation and is an alternative to plain radiographs for imaging the spine. However, it is performed in the supine position; consequently, the angle measurements are underestimated compared to standing X-rays, due to the effects of gravity. Several studies have examined the relationship in spinal alignment between standing plain X-rays and supine MR images [6-8]. Wessberg et al. [6] found that supine axially loaded MRI correlated well with standing Cobb angle measurements. Lee et al. [7] demonstrated that unloaded spine MRI could reliably be translated into equivalent radiographic measures with an acceptable range of error in adolescent idiopathic scoliosis. Wang et al. [8] found that supine MRI was a valid alternative to standing X-rays for measuring upper thoracic kyphosis in the sagittal plane. However, no study has addressed the use of MRI for evaluating spinal sagittal alignment in degenerative lumbar disease.

Therefore, this study compared standing plain X-rays and supine MRI for evaluating spinal sagittal alignment in degenerative lumbar disease, and examined the possible correlation between measurements 
made using the two imaging modes.

\section{Material And Methods}

Patients with a diagnosis of degenerative lumbar disease, who had a standing plain X-ray and MRI performed between January 1, 2015 and June 1, 2016, were included in this study. The Institutional Review Board approved this study, and each patient signed a written consent form. The inclusion criteria were as follows: patients with lumbar canal stenosis, lumbar disc hernia or lumbar spondylolisthesis; age $\geq 50$ years; no underlying congenital or neurological abnormalities; no leg length discrepancy $>2 \mathrm{~cm}$; Cobb angle $<20^{\circ}$; and no more than 1 month between the standing X-ray and MRI. The standing radiographs were taken with a Philips MRI machine (Philips Healthcare, Andover, MA, USA), and the MRI was obtained with a 1.5 T MRI scanner (Siemens, Germany). All of the digital images were downloaded and archived on a picture archiving and communication system (PACS).

The radiological examination protocol was standardized for all of the participants. For each participant, standing anteroposterior and left lateral $X$-rays covering the spine and pelvis were obtained. The supine MRI covered the region from T10 to the coccyx. The Cobb angle and spinal sagittal parameters measurements for both imaging modes were obtained using the angle measurement tools provided by the PACS. On MR images, the Cobb angle was calculated on the T1-weighted coronal plane cut that included the clearest view of the endplates of the end vertebrae of the curve. The same end vertebrae were used to measure the Cobb angle on the lateral plain X-rays and MR images. The thoracolumbar junction kyphosis (TJK) was measured from the superior end plate of T10 to the inferior end plate of L2. Lumbar lordosis (LL) was measured from the inferior end plate of T12 to the superior end plate of S1 using the Cobb method. Sacral slope (SS) was the angle between the superior end plate of S1 and a horizontal line. We could not calculate the pelvic incidence (PI) or pelvic tilt (PT) because the lumbar MRI did not include the femoral heads.

\section{Results}

The study enrolled 64 patients ( 30 males, 34 females; average age, $62 \pm 7$ years; mean body mass index, $23.79 \pm 3.1 \mathrm{~kg} / \mathrm{m}^{2}$ ). Thirty-three patients were diagnosis of lumbar stenosis; twenty patients were diagnosis of lumbar disc hernia; and eleven patients were diagnosis of lumbar spondylolisthesis. The average time between when the plain radiographs and supine MRI was taken was $1 \pm 1$ week. The average TJK measured from the radiographs and MR images was $11 \pm 9^{\circ}$ and $9 \pm 7^{\circ}$, respectively; the average $L L$ was $40 \pm 16^{\circ}$ and $40 \pm 13^{\circ}$; and the average SS was $33 \pm 11^{\circ}$ and $35 \pm 9^{\circ}$. These results suggest that the TJK measured from the MR images tended to underestimate the radiographic measurements by $2^{\circ}$, whereas the SS measurements obtained from the MR images tended to overestimate the radiographic measures by $2^{\circ}$. The $L L$ measurements were roughly equal with both imaging methods. Evaluating the relationship between the MRI and radiographic measurements, the Pearson coefficient was $r=0.85$ for TJK, $r=0.785$ for $L L$, and $r=0.654$ for SS. The X-ray and MRI measurements showed roughly linear relationships (Table 1; Figs. 1-3). 
Table 1

Trend equations of $\mathrm{X}$-ray and MRI

\begin{tabular}{|lll|}
\hline & Best-Fit Equation & Absolute Error $\left({ }^{\circ}\right)$ \\
\hline TJK & X-ray $=1.076 \mathrm{MRI}+1.632$ & 4.575 \\
LL & X-ray $=0.973 \mathrm{MRI}+1.877$ & 10.1 \\
SS & X-ray $=0.822 \mathrm{MRI}+3.941$ & 8.227 \\
\hline
\end{tabular}

The intra-observer reliabilities of the TJK, LL, and SS measurements obtained from MRI ranged from 0.965 to 0.972 (Table 2), indicating that the results are in strong to near-perfect agreement. In contrast, the intra-observer reliabilities obtained from the X-rays ranged from 0.800 to 0.943 (Table 2). The interobserver reliability quantified using intra-class correlation coefficients (ICCs) was 0.713 to 0.856 for Xrays and 0.891 to 0.925 for MRI, suggesting strong agreement (Table 3 ).

Table 2

Intraobserver reliability for the 2 observers.

\begin{tabular}{|ccccccc|}
\hline \multicolumn{3}{|c}{ Observer1 } & \multicolumn{2}{l}{ Observer2 } & \multicolumn{2}{l|}{ Overall } \\
\hline & X-ray & MRI & X-ray & MRI & X-ray & MRI \\
\hline TJK & 0.956 & 0.968 & 0.923 & 0.971 & 0.943 & 0.970 \\
\hline LL & 0.856 & 0.972 & 0.869 & 0.952 & 0.860 & 0.965 \\
\hline SS & 0.815 & 0.961 & 0.773 & 0.978 & 0.800 & 0.972 \\
\hline
\end{tabular}

Table 3

Interobserver reliability of the measurements from standing X-ray and supine MRI

\begin{tabular}{|llll|}
\hline & TJK & LL & SS \\
\hline X-ray & 0.782 & 0.856 & 0.713 \\
\hline MRI & 0.891 & 0.925 & 0.910 \\
\hline
\end{tabular}

\section{Discussion}

The standing X-ray, which spinal surgeons use to plan surgery, is considered the gold standard for measuring spinal alignment [9]. However, repeated doses of ionizing radiation increases the risk of health problems, such as an altered immune response and increased cancer risk $[5,10]$. Although improvements in radiographic technology have minimized radiation exposure, long-term health complications remain problematic $[11,12]$. Thus, methods that do not use radiation are needed to evaluate spinal alignment. 
In this study, we found that the correlation between the standing radiographic and supine MRI measurements of SS, TJK, and LL were very good in degenerative lumbar disease. Several studies have explored the relationship between Cobb's angle measured from standing radiographs and supine MRI. Wessberg et al. [6] used an axial load device to adjust for the unloaded spinal condition present in supine MR images, and found a near-linear relationship between the plain radiographic and MRI Cobb measures. Lee et al. [7] also found a near-linear relationship without additional axial loading, although supine MRI tended to underestimate plain radiographs by $10^{\circ}$ on average in adolescent idiopathic scoliosis. For sagittal balance, Bernstein et al. [13] observed no significant difference between thoracic kyphosis angles measures from plain radiographs and MRI. However, the authors pointed out that these results cannot be transferred to lumbar lordosis measurement. Wang et al. [8] compared supine MR imaging and standing X-rays in the evaluation of the sagittal alignment of the upper thoracic spine, and found no significant difference in the sagittal angles. However, all of the previous studies were performed in patients with adolescent idiopathic scoliosis. To date, no study has compared spinal alignment on standing plain radiographs and supine MRI in patients with degenerative lumbar disease.

This study found that the measurements of SS, TJK, and LL from supine MRI were strongly correlated with those from standing $X$-rays ( $r=0.85$ for TJK, $r=0.785$ for $L L$, and $r=0.654$ for SS). The coefficient for SS indicated a moderate correlation, which may be due to the poor visibility of the S1-vertebra in standing X-rays [13]. The LL measures obtained from MRI and X-rays were approximately equal. LL is important in lumbar degenerative disease. When it is significantly reduced, sagittal plane imbalance occurs. Then flat back syndrome can develop, which is characterized by back pain, a stooped posture, and an impaired gait[14]. The restoration of lumbar lordosis is important in the preoperative plan. According to our results, supine MRI can be used in the evaluation of spinal sagittal alignment without unnecessary exposure to radiation.

The S1 vertebra is frequently obscured on X-rays by the overlying ilium; thus, more than $50 \%$ of the S1 vertebrae cannot be viewed properly in X-rays [13]. We found that the ICC for intra-observer reliability increased from 0.800 (standing X-ray) to 0.972 (MRI) because MRI provided a clearer picture of the vertebrae. Therefore, MRI measurements enable more precise sagittal measurements.

\section{Conclusion}

In conclusion, supine MRI can be directly translated into sagittal alignment angle measures obtained from standing X-rays with an acceptable degree of accuracy. MRI avoids the impaired visibility caused by the overlapping ilium, while reducing the patient's exposure to radiation.

\section{Abbreviations}

MRI: magnetic resonance imaging; TJK:thoracolumbar junction kyphosis; LL:Iumbar lordosis; SS:sacral slope; Pl:pelvic incidence; PT:pelvic tilt 


\section{Declarations \\ Copeting interests}

The authors declare that they have no competing interests.

\section{Authors' contributions}

W. collected, analyzed, and interpreted the patient data and was a major contributor in writing the manuscript.

J. B. and Z. P. analyzed and interpreted the patient data and searched literatures. D. X. designed the study and wrote the manuscript. All authors read and approved the final manuscript.

\section{Ethics approval and consent to participate}

This study was approved by the institutional review board of 2nd Affiliated Hospital, School of Medicine, Zhejiang University. Written informed consent was obtained from each patient.

\section{Consent for publication}

Not applicable.

\section{Availability of data and materials}

All data generated or analyzed during this study are included in this published article.

\section{Funding}

There is no funding source for this study.

\section{Acknowledgements}

None.

\section{References}

1. Diebo BG, Henry J, Lafage V, Berjano P. Sagittal deformities of the spine: factors influencing the outcomes and complications. Eur Spine J. 2015;24(Suppl 1):3-15. 
2. Sabou S, Tseng TH, Stephenson J, Siddique I, Verma R, Mohammad S. Correction of sagittal plane deformity and predictive factors for a favourable radiological outcome following multilevel posterior lumbar interbody fusion for mild degenerative scoliosis. Eur Spine J. 2016;25(8):2520-6.

3. Boulay C, Tardieu C, Hecquet J, Benaim C, Mouilleseaux B, Marty C, Prat-Pradal D, Legaye J, DuvalBeaupere G, Pelissier J. Sagittal alignment of spine and pelvis regulated by pelvic incidence: standard values and prediction of lordosis. European spine journal: official publication of the European Spine Society the European Spinal Deformity Society the European Section of the Cervical Spine Research Society. 2006;15(4):415-22.

4. Doody MM, Lonstein JE, Stovall M, Hacker DG, Luckyanov N, Land CE. Breast cancer mortality after diagnostic radiography: findings from the U.S. Scoliosis Cohort Study. Spine (Phila Pa 1976). 2000;25(16):2052-63.

5. reTRRao PS, Gregg EC. A revised estimate of the risk of carcinogenesis from x-rays to scoliosis patients. Invest Radiol. 1984;19(1):58-60.

6. Wessberg P, Danielson BI, Willen J. Comparison of Cobb angles in idiopathic scoliosis on standing radiographs and supine axially loaded MRI. Spine (Phila Pa 1976). 2006;31(26):3039-44.

7. Lee MC, Solomito M, Patel A. Supine magnetic resonance imaging Cobb measurements for idiopathic scoliosis are linearly related to measurements from standing plain radiographs. Spine (Phila Pa 1976). 2013;38(11):E656-61.

8. Wang F, Sun X, Mao S, Liu Z, Qiao J, Zhu F, Zhu Z, Pan T, Chen L, Zhao Q, et al. MRI May Serve as a Valid Alternative to Standing Radiography in Evaluating the Sagittal Alignment of the Upper Thoracic Spine. Clin Spine Surg. 2017;30(3):124-8.

9. Bess S, Protopsaltis TS, Lafage V, Lafage R, Ames CP, Errico T, Smith JS. International Spine Study G: Clinical and Radiographic Evaluation of Adult Spinal Deformity. Clin Spine Surg. 2016;29(1):6-16.

10. Buitenhuis W, Fritschi L, Thomson A, Glass D, Heyworth J, Peters S. Occupational exposure to ionizing radiation and risk of breast cancer in Western Australia. J Occup Environ Med. 2013;55(12):1431-5.

11. Sunavala-Dossabhoy G, Palaniyandi S, Richardson C, De Benedetti A, Schrott L, Caldito G. TATmediated delivery of Tousled protein to salivary glands protects against radiation-induced hypofunction. Int J Radiat Oncol Biol Phys. 2012;84(1):257-65.

12. Pacheco R, Stock H. Effects of radiation on bone. Curr Osteoporos Rep. 2013;11(4):299-304.

13. Bernstein P, Hentschel S, Platzek I, Zwingenberger S, Weigel S, Huhne S, Seifert J. The assessment of the postoperative spinal alignment: MRI adds up on accuracy. Eur Spine J. 2012;21(4):733-8.

14. Hsu HT, Yang SS, Chen TY. The Correlation Between Restoration of Lumbar Lordosis and Surgical Outcome in the Treatment of Low-grade Lumbar Degenerative Spondylolisthesis With Spinal Fusion. Clin Spine Surg. 2016;29(1):E16-20.

\section{Figures}




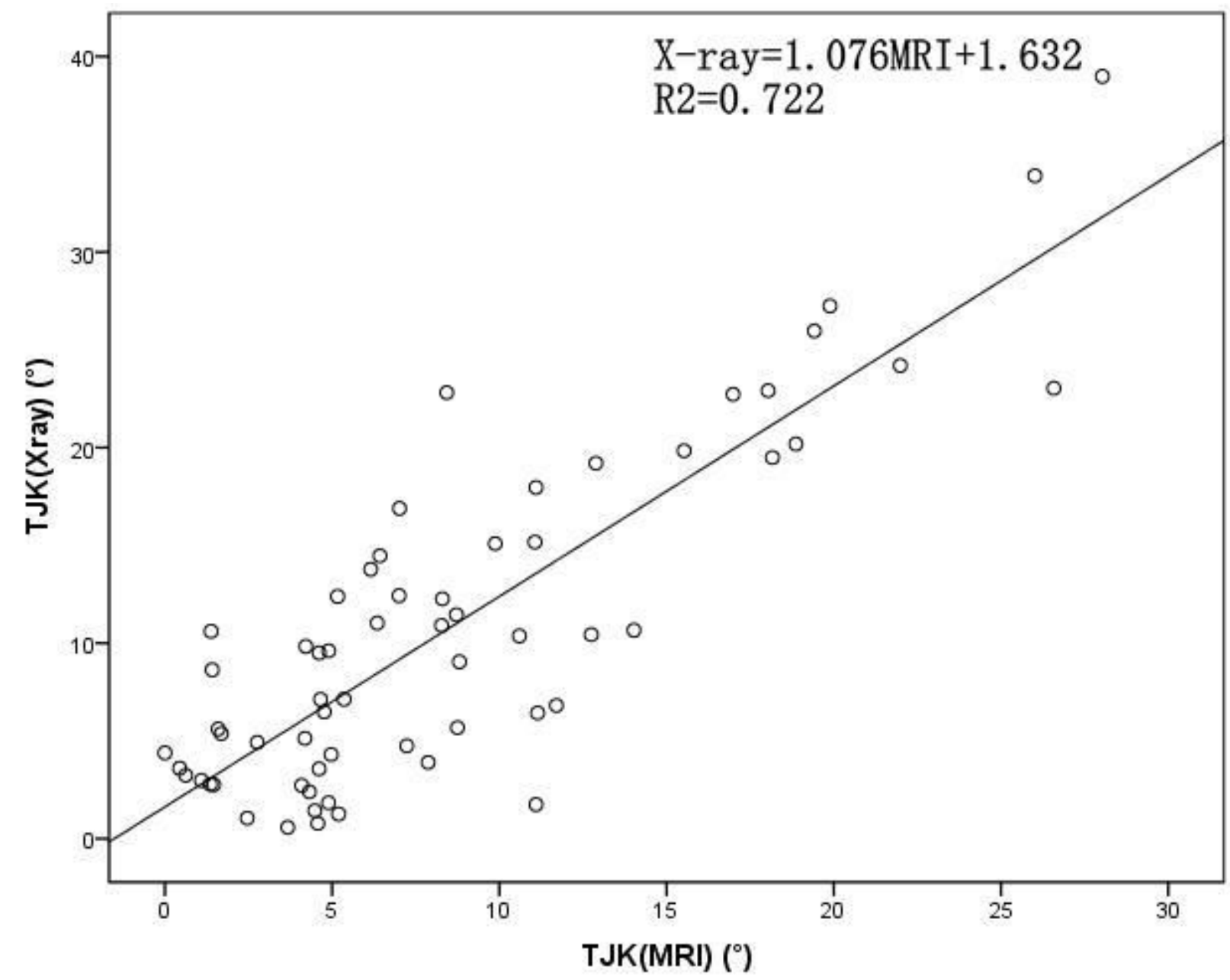

Figure 1 


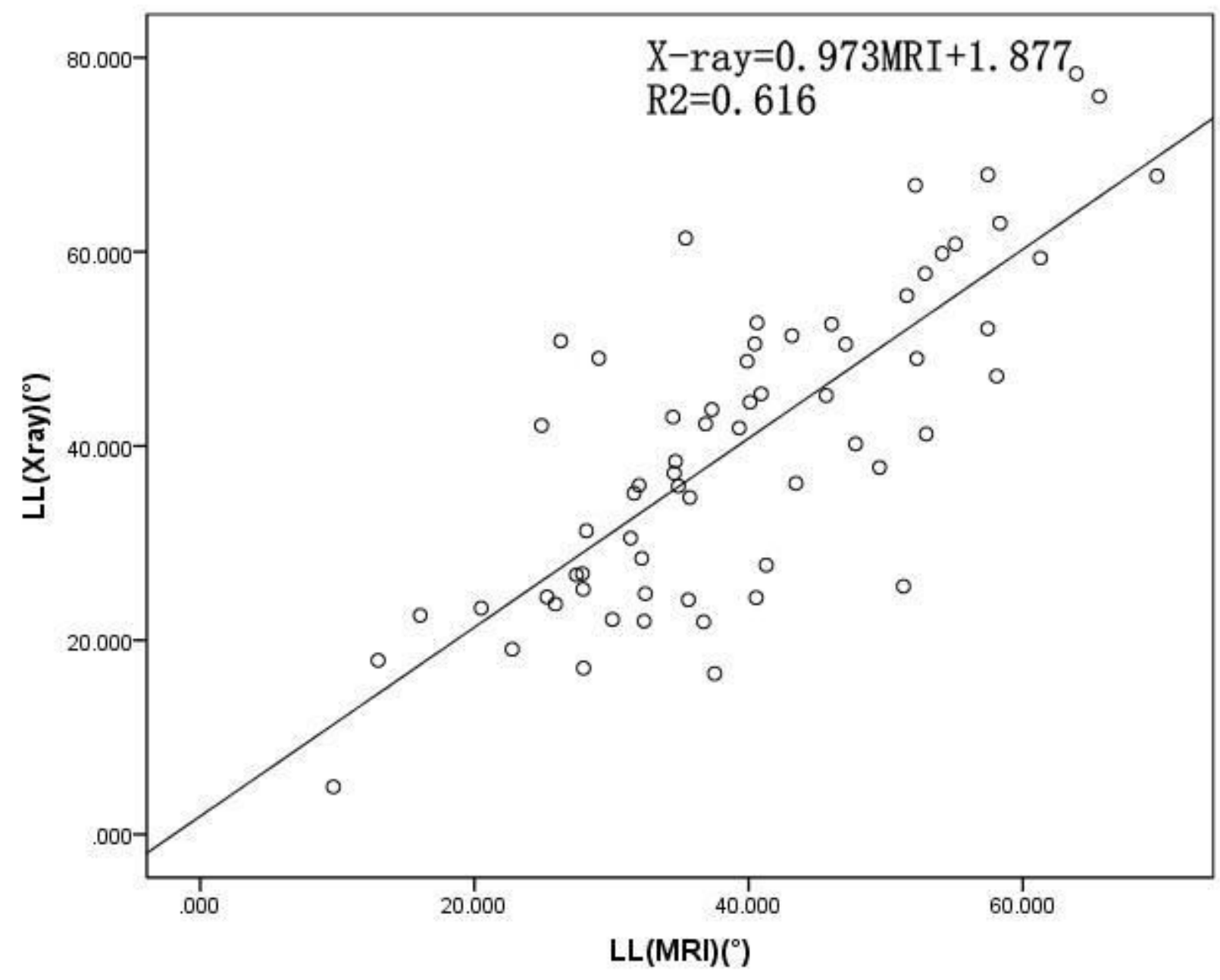

Figure 2 


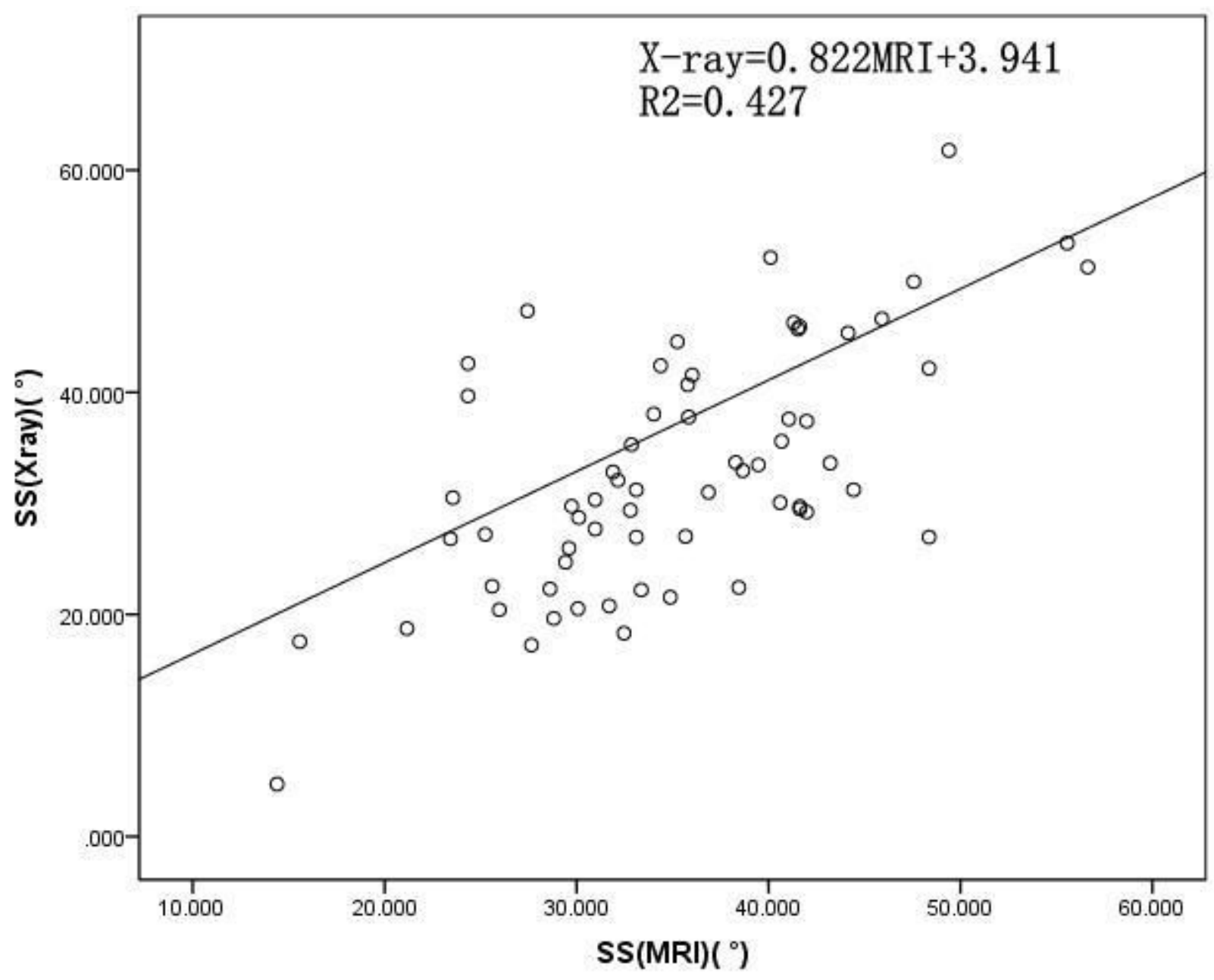

Figure 3 